\title{
The EGL-3 Proprotein Convertase Regulates Mechanosensory Responses of Caenorhabditis elegans
}

\author{
Jamie Kass, ${ }^{1,2}$ Tija C. Jacob, ${ }^{2}$ Peter Kim, ${ }^{1}$ and Joshua M. Kaplan ${ }^{2}$ \\ ${ }^{1}$ Department of Molecular Biology, Massachusetts General Hospital and Department of Genetics, Harvard Medical \\ School, Boston, Massachusetts 02114, and 2Department of Molecular and Cell Biology, University of California, Berkeley, \\ California 94720-3200
}

\begin{abstract}
Neuroactive peptides are packaged as proproteins into dense core vesicles or secretory granules, where they are cleaved at dibasic residues by copackaged proprotein convertases. We show here that the Caenorhabditis elegans egl-3 gene encodes a protein that is $57 \%$ identical to mouse proprotein convertase type 2 (PC2), and we provide evidence that this convertase regulates mechanosensory responses. Nose touch sensitivity (mediated by ASH sensory neurons) is defective in mutants lacking GLR-1 glutamate receptors (GluRs); however, muta-
\end{abstract}

tions eliminating the egl-3 PC2 restored nose touch sensitivity to g/r-1 GluR mutants. By contrast, body touch sensitivity (mediated by the touch cells) is greatly diminished in egl-3 PC2 mutants. Taken together, these results suggest that egl-3 PC2processed peptides normally regulate the responsiveness of $C$. elegans to mechanical stimuli.

Key words: mechanosensation; neuropeptide; synapse; proprotein convertase; egl-3; glr-1; glutamate receptor; C. elegans
Sensory systems adapt to changes in environmental inputs. The smallest detectable change in a stimulus often varies depending on the level of ambient signaling. Persistent sensory stimulation can enhance, diminish, or eliminate sensitivity to a particular stimulus. For example, type C nociceptors (which sense painful stimuli) often have enhanced sensitivity after a previous injurious stimulus. Conversely, persistent exposure to odorants typically diminishes or eliminates the response of olfactory neurons. Regulation of sensory responsiveness allows animals to produce adaptive changes in behavior in response to changing ambient conditions.

Secretion of neuromodulatory peptides has often been proposed as a mechanism for producing adaptive changes in sensory responses (Kupfermann, 1991). At synapses, neuropeptides are released as cotransmitters with other classical neurotransmitters. Neuropeptides are thought to modulate the impact of the coreleased classical neurotransmitters (Kupfermann, 1991). For example, neuropeptides can act presynaptically to facilitate or inhibit release of other neurotransmitters. Alternatively, neuropeptides can modify the sensitivity of the postsynaptic cell to released neurotransmitters. Alterations in synaptic efficacy produced by neuroactive peptides could provide a common mechanism for modulating sensory responses.

Proprotein convertases comprise a gene family that currently consists of seven members in mammals (PC1-PC7). Several proprotein convertases have been genetically characterized. Muta-

Received March 21, 2001; revised Sept. 18, 2001; accepted Sept. 18, 2001.

This work was supported by National Institutes of Health Grant NS32196 to J.K. T.C.J. is a Howard Hughes predoctoral fellow. We thank the following for advice, strains, and reagents: M. Grunwald, I. Dickerson, S. Pierce, G. Ruvkun, C. Li, A. Hart, A. Fire, B. Harfe, The C. elegans Genetic Stock Center, A. Coulson, and members of the Kaplan laboratory. We especially thank Axys Pharmaceuticals (South San Francisco, CA) for providing the $n r 2090$ allele.

Correspondence should be addressed to Dr. Joshua M. Kaplan, LSA 361, University of California, Berkeley, CA 94720-3200. E-mail: Joshkap@socrates.berkeley.edu.

J. Kass's present address: Virtual Text, 1100 Massachusetts Avenue, Cambridge, MA 02138

Copyright (ㄷ) 2001 Society for Neuroscience $\quad 0270-6474 / 01 / 219265-08 \$ 15.00 / 0$ tions in human PC1 have been associated with obesity and accumulation of proinsulin (Jackson et al., 1997). Mice lacking PC2 or a chaperonin required for production of active PC2 (7B2) accumulate proinsulin, proglucagon, and prosomatostatin (Furuta et al., 1997; Westphal et al., 1999). Absence of the Drosophila amontillado gene (orthologous to PC2) causes a defect in hatching behavior (Siekhaus and Fuller, 1999). Thus, although a great deal is known about endocrine defects observed in neuropeptide biosynthetic mutants, much less is known about the effect of these mutations on behavioral circuits.

Here we show that the Caenorhabditis elegans ortholog of proprotein convertase type 2 (PC2) is encoded by the egl-3 gene and that the egl-3 $\mathrm{PC} 2$ regulates responsiveness to mechanical stimuli.

\section{MATERIALS AND METHODS}

\section{Screen and genetic mapping of glr-1 suppressors}

Hermaphrodite $g l r-1(n 2461)$ worms were mutagenized with $0.1 \mathrm{M}$ ethyl methanesulfonate for $4 \mathrm{hr}$ at $20^{\circ} \mathrm{C}$. F2 progeny of mutagenized worms were tested for response to nose touch. From 13,000 F2 animals, one mutant, nu349, was isolated. The egl-3(nr2090) deletion allele was isolated by Axys Pharmaceuticals (South San Francisco, CA) (Liu et al., 1999).

Three factor mapping data for the nu349 egg-laying defective phenotype were as follows: dpy-11(e224) (19/19) (unc-42(e270) nu349) and unc-42(e270) (6/13) nu349 (7/13) sma-1(e30). This placed egl-3(nu349) between unc-42 and sma-1, a 1.3 centimorgan interval.

\section{Transgenes and germline transformation}

Plasmids were constructed by standard techniques, and sequences were verified where appropriate; full details are available on request. Transgenic strains were isolated by microinjecting various plasmids (typically at either 50 or $100 \mathrm{ng} / \mu \mathrm{l})$ using $t t x-3:: g f p$ (O. Hobert, Columbia Presbyterian, New York, NY) as a marker. Plasmids and transgenic strains were constructed as follows:

For rescue experiments. Initially a pool of four cosmids (ZC273, F21F2, C26B6, and C04G9) from the candidate region were microinjected into glr-1(n2461);egl-3(nu349) double mutants. Three transgenic lines were obtained, and two were rescued for the egg-laying (Egl), body touch sensitivity (Mec), and nose touch sensitivity (Not) phenotypes. Subsequently, transgenes containing only C26B6 were isolated, and 8/12 trans- 
genic lines were rescued for Egl, Mec, and Not phenotypes. No rescue was obtained with transgenes containing ZC273, F21F2, or C04G9.

EGL-3 constructs. Two CELPC2 plasmid subclones were subsequently shown to rescue all behavioral defects caused by egl-3(nu349). KP\#451 contains a $6.96 \mathrm{~kb}$ SpeI fragment, spanning nucleotides 13140-20103 of C26B6. KP\#452 contains a $10.5 \mathrm{~kb}$ Pst I fragment, spanning nucleotides 10871-21418 of C26B6. KP\#452 contains $4.2 \mathrm{~kb}$ of $5^{\prime}$ and $2.3 \mathrm{~kb}$ of $3^{\prime}$ flanking sequences. KP\#454 contains a rescuing green fluorescent protein (GFP)-tagged egl-3 construct, in which GFP was fused in-frame at the $\mathrm{C}$ terminus of the $10.5 \mathrm{~kb}$ egl-3 genomic construct. Transgenes containing KP\#454 also rescue the Egl, Not, and Mec phenotypes caused by egl-3(nu349). KP\#508 contains an osm-10 promoter sequence, which is expressed in ASH, ASI, PHA, and PHB (Hart et al., 1999), driving expression of the egl-3 genomic construct. KP\#509 contains a $g l r-1$ promoter sequence, which is expressed in the command neurons (Hart et al., 1995; Maricq et al., 1995), driving expression of the egl-3 genomic construct.

\section{Sequencing of egl-3 alleles}

Sequence changes in mutant alleles were determined by amplifying exons and exon-intron boundaries from mutant strains and direct sequencing of the amplified products by cycle sequencing. Mutations found in each allele are indicated in Figure 3.

\section{Analysis of behaviors and drug sensitivities}

Nose touch, osmotic avoidance, and volatile repellent assays were performed as previously described (Kaplan and Horvitz, 1993; Troemel et al., 1995; Hart et al., 1999). Behaviors were quantified as follows: nose touch avoidance (Not), percentage of trials in which animals responded to touch with an eyelash by stopping forward movement or reversing; osmotic avoidance (Osm), percentage of animals that escape a ring of 8 M glycerol in $<10 \mathrm{~min}$; volatile avoidance (Sos), average time to initiate backward movement in response to an eyelash dipped in 1-octanol; and body touch sensitivity, percentage of trials where animals responded to touch to the anterior body region with an eyelash.

Drugs were purchased from Sigma (St. Louis, MO). Egg-laying assays were performed as described (Trent et al., 1983), with a few modifications. Drug treatments were as follows (in milligrams per milliliter): serotonin 3 and imipramine 0.75 . To test for sensitivity to a particular drug, three hermaphrodites were placed in a microtiter well containing a solution of the drug dissolved in M9 buffer, and, at various times after transfer, the number of eggs in each well was counted. Final counts were taken at $60 \mathrm{~min}$ for serotonin and $90 \mathrm{~min}$ for imipramine. Each assay was performed in triplicate.

To determine the number of eggs in the uterus, fourth larval stage hermaphrodites were placed at $15^{\circ} \mathrm{C}$ for $\sim 18 \mathrm{hr}$. Individual hermaphrodites were placed on an agar dish, and their cuticles were dissolved by application of bleach. The number of eggs in the uterus could then be counted as observed under a dissecting microscope.

\section{Laser operations}

ASH neurons in first stage larvae were identified based on their nuclear position and morphologies and were killed with a laser microbeam (Laser Sciences Inc., Newton, MA) as described previously (Kaplan and Horvitz, 1993). Nose touch assays were conducted on animals when they reached the young adult stage, $3 \mathrm{~d}$ after surgery, by an experimenter unaware of the animal's genotype or operative status. After the behavioral testing, animals were stained with the dye $\mathrm{DiO}$, to confirm that both ASH neurons had been killed.

\section{Antibodies, immunostaining, and GFP reporters}

Anti-serotonin antibodies were purchased from H. Steinbusch (University of Limburg, Maastricht, The Netherlands). Anti-Phe-Met-Arg-Phe amide (FMRFamide) antibodies were a gift from Chris Li (Boston University, Boston, MA). A synthetic peptide [(C)SRRPKDDDSKDG FTNWP-COOH] corresponding to amino acids 565-581 of the predicted EGL-3 sequence was conjugated to keyhole limpet hemocyanin and injected into rabbits. Peptide synthesis, conjugation, injections, and bleeds were performed by Zymed Laboratories (South San Francisco, CA). Anti-EGL-3 antibodies were affinity-purified from crude serum with peptide conjugated to SulfoLink Coupling Gel (Pierce, Rockford IL), according to the recommendation of the manufacturer. A glutathione $S$-transferase fusion protein containing the ecto domain of the GLR-1 glutamate receptor was used to raise anti-GLR-1 antibodies in

\section{A Mechanosensory Cells}
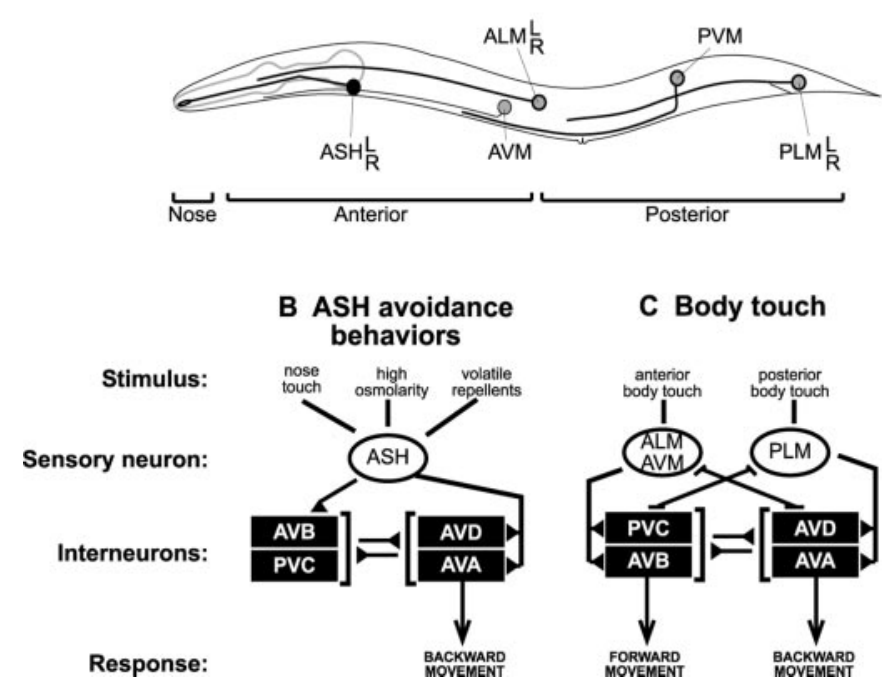

Figure 1. Mechanosensory circuits for nose and body touch responses. A, Nose touch stimuli evoke backward locomotion and are sensed by ASH neurons (black circle). ASH neurons have dendritic processes that are exposed to the external environment through the amphid pore at the tip of the worm's nose (Perkins et al., 1986). Body touch sensitivity is mediated by six touch cells ( gray circles), which have sensory endings in either the anterior or posterior body regions. Touch to the anterior half of the body elicits backward locomotion and vice versa for posterior touch stimuli (Chalfie and Au, 1989). B, C, Synaptic connections underlying the nose and body touch responses are illustrated. Chemical synapses are indicated by lines with black triangles, and gap junctions by lines with bars. $B$, Nose touch responses are primarily mediated by the ASH neurons. Two other sensory neurons also contribute to this response but quantitatively account for $<40 \%$ of the normal responsiveness (Kaplan and Horvitz, 1993). For simplicity, these minor nose touch sensory neurons are not shown here. The ASH neurons also mediate responsiveness to hyperosmolarity and volatile repellents (Bargmann et al., 1990; Troemel et al., 1995). All ASH stimuli evoke backward movement via synapses between ASH and the command neurons for locomotion (AVA, AVB, and AVD). The glr-1 GluRs are clustered at command neuron synapses (Rongo et al., 1998). C, Body touch responses are mediated by the touch cells. Touch cells form gap junctions and chemical synapses with the command neurons. The touch cell-command neuron synapses are thought to be inhibitory (Chalfie et al., 1985; Wicks and Rankin, 1995; Wicks et al., 1996) and glutamatergic (Lee et al., 1999). Adapted from Kaplan and Horvitz (1993) and Driscoll and Kaplan (1997).

rats. Whole-mount immunofluorescence of fixed worms was done using Bouin's fixative, as described (Nonet et al., 1997). Egg-laying muscle morphology was observed in egl-3(nu349) mutants using an integrated transgene containing $\mathrm{pBH} 10.21$, a GFP reporter expressed in the egglaying muscles (Harfe and Fire, 1998).

\section{RESULTS}

We have used a genetic strategy to investigate how two particular C. elegans mechanosensory responses, aversive responses to body touch and nose touch, are modulated. Nose and body touch responses are mediated by different classes of mechanosensory neurons (Fig. $1 A$ ). Body touch sensitivity is mediated by a set of six mechanosensory neurons, termed the touch cells, which have sensory endings in either the anterior or posterior body regions (Chalfie and $\mathrm{Au}, 1989$ ). Anterior body touch evokes backward movement, whereas posterior body touch evokes forward movement. Nose touch sensitivity is primarily mediated by the ASH sensory neurons (Kaplan and Horvitz, 1993). The ASH neurons also mediate responsiveness to hyper-osmolarity and to volatile repellents (Bargmann et al., 1990; Troemel et al., 1995). In this 
respect, the $\mathrm{ASH}$ neurons are similar to mammalian type $\mathrm{C}$ nociceptor neurons, which are also polymodal, sensing painful chemical, thermal, and mechanical stimuli (Besson and Chaouch, 1987). All three ASH sensory stimuli evoke backward movement but do so via distinct signaling pathways (Hart et al., 1999). The effects of nose and body touch on locomotion are mediated by direct synaptic connections between mechanosensory neurons and interneurons that drive locomotion, which are referred to here as command neurons (Fig. $1 B, C$ ).

Several results suggest that ASH-command neuron synapses are glutamatergic. All three ASH-mediated sensory behaviors are defective in eat-4 mutants (Berger et al., 1998; Hart et al., 1999), which lack the vesicular glutamate transporter (VGLUT) (Bellochio et al., 2000; Takamori et al., 2000), and hence are defective for glutamate neurotransmission (Lee et al., 1999). The eat-4 VGLUT gene is expressed in the ASH neurons (Lee et al., 1999), suggesting that ASH is glutamatergic. Consistent with this idea, the $g l r-1$ gene encodes an AMPA-type glutamate receptor (GluR) that is expressed in the command interneurons (Hart et al., 1995; Maricq et al., 1995), and these receptors are clustered at command neuron synapses in vivo (Rongo et al., 1998). Mutants that lack glr-1 GluRs (Hart et al., 1995; Maricq et al., 1995) or those that fail to cluster $g l r-1$ GluRs at command neuron synapses (Rongo et al., 1998) are defective for ASH-mediated nose touch sensitivity. Together these results demonstrate that glutamate neurotransmission is required for ASH-mediated sensory responses and that ASH-command neuron synapses are glutamatergic.

\section{The nu349 mutation restores nose touch sensitivity to glr-1 GluR mutants}

To identify genes that modulate ASH sensory responses, we screened for mutations that restored nose touch responsiveness to homozygous glr-1 GluR mutants. Our strategy was based on the following rationale. The glr-1 GluRs are required for ASHmediated nose touch responses but are not required for ASHmediated osmosensory responses (Hart et al., 1995; Maricq et al., 1995). These results suggested that a nose touch stimulus is insufficient to evoke an efficacious signal at ASH-command neuron synapses in $g l r-1$ GluR mutants, although these same synapses retain the ability to mediate responses to another stimulus. Therefore, we reasoned that mutations that facilitate signaling at ASH-command neuron synapses would restore nose touch sensitivity to $g l r-1$ GluR mutants.

Approximately 6500 haploid genomes were screened, and the mutant nu349 was isolated as a suppressor of the nose touch insensitivity of $g l r-1(n 2461)$ mutants. The nu349 mutation completely restored the nose touch sensitivity of both $\operatorname{glr}-1(n 2461)$ and of $g l r-1(k y 176)$ mutants (Fig. 2A), which correspond to a premature stop codon and a deletion allele, respectively (Hart et al., 1995; Maricq et al., 1995). The nu349 mutation also restored nose touch sensitivity to lin-10 mutants (Fig. $2 A$ ). Although the nose touch sensitivity of lin-10; nu349 double mutants was restored, glr-1 GluRs still failed to cluster at command neuron synapses in these animals (data not shown). These results suggest that the nu349 mutation functionally bypassed the requirement for $g l r-1$ GluRs in the ASH-mediated nose touch response.

\section{The egl-3 gene encodes a PC2}

We mapped nu349 to the right arm of chromosome $\mathrm{V}$, between unc-42 and sma-1. In addition to $g l r-1$ suppression, homozygous nu349 mutants had two additional behavioral phenotypes: defec-
A
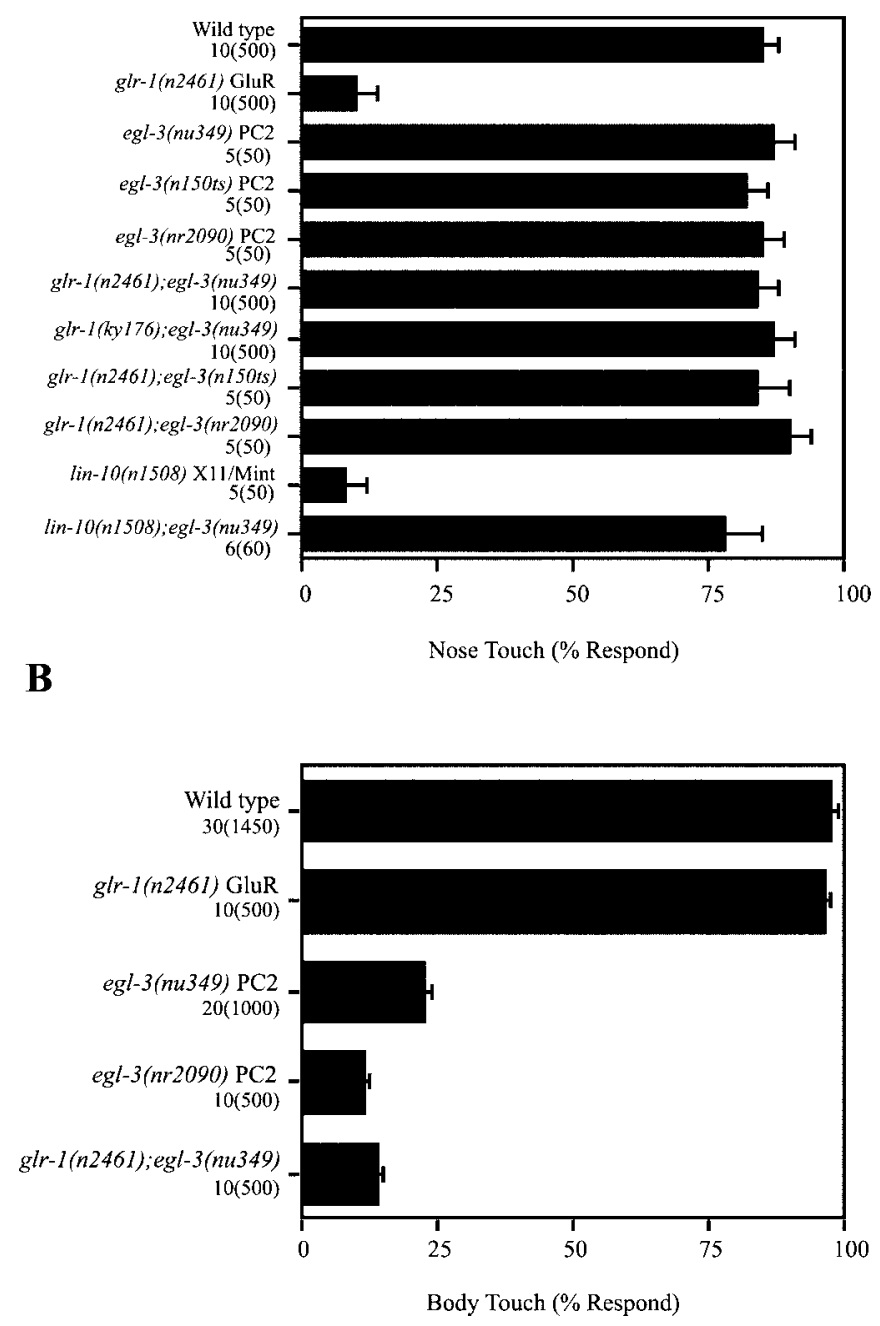

Figure 2. Restoration of the nose touch response by egl-3 PC2 mutations. Nose and body touch responses of several strains were compared, as detailed in Materials and Methods. $A$, The egl-3(nu349) PC2 allele restored full nose touch responsiveness to two different $g l r-1$ GluR mutants: n2461 a premature stop codon, and ky176 a deletion allele (Hart et al., 1995; Maricq et al., 1995). The egl-3(nu349) PC2 allele also restored nose touch responsiveness to lin-10 mutants, which fail to cluster $g l r-1$ GluRs at sensory-command neuron synapses. Two other egl-3 PC2 alleles (n150ts and $n r 2090)$ also restored nose touch responsiveness to glr-1 GluR mutants. These results suggest that egl-3 PC2 mutations functionally bypass the requirement for $g l r-1$ GluRs at ASH-command neuron synapses. $B$, Body touch responsiveness was reduced in homozygous egl-3 PC2 mutants. Values shown are mean \pm SEs. The numbers listed below each genotype indicate the number of animals and trials, as follows: number of animals(number of trials).

tive egg-laying (Egl) (see Materials and Methods for details) and insensitivity to body touch (Mec) (Tables 1, 2, Fig. 2B). Therefore, we examined other egg-laying and mechanosensory genes in this region and found that $n u 349$ was an allele of egl-3, which was originally identified in a screen for egg-laying defective mutants (Trent et al., 1983). Other egl-3 alleles also restored nose touch sensitivity to glr-1 GluR mutants (Fig. 2A), and the nu349 mutation failed to complement other egl-3 alleles for the egg-laying defect (detailed below).

We cloned the egl-3 gene based on its genetic map position and 


\begin{tabular}{llll}
\hline \multicolumn{3}{l}{ Table 1. CELPC2 transgenes rescue egl-3 } & PC2 mutant phenotypes \\
Genotype & Transgene & Nose touch & Body touch \\
\hline Wild type & None & 85 & $\mathrm{WT}$ \\
glr-1(n2461) & None & 10 & $\mathrm{WT}$ \\
glr-1(n2461);egl-3(nu349) & None & 85 & $\mathrm{Mec}$ \\
glr-1(n2461);egl-3(nu349) & C26B6 & 10 & $\mathrm{WT}$ \\
glr-1(n2461);egl-3(nu349) & C51E3 & 12 & $\mathrm{WT}$ \\
glr-1(n2461);egl-3(nu349) & CELPC2 & 1 & $\mathrm{WT}$
\end{tabular}

Transgenic strains were isolated, and behaviors were quantitated as described in Materials and Methods. The cosmid clones C26B6 and C51E3 span the CELPC2 gene. The CELPC2 refers to KP\#452, a $10.55 \mathrm{~kb}$ CELPC2 genomic fragment. Rescuing activity of a transgene (in a glr-1;egl-3 double mutant) is indicated by diminishing the nose touch response and restoration of the body touch response. Rescue of the egg-laying defect is described in Results. Mec indicates a diminished response to body touch. WT indicates wild-type response. Values reported are means.

found that it corresponds to CELPC2, a previously described $C$. elegans ortholog of the proprotein convertase PC2 (GomezSaladin et al., 1994). Proprotein convertases are endoproteases that mediate processing of prohormones and proneuropeptides (Creemers et al., 1998; Steiner, 1998). Transgenes containing either cosmids (C26B6 or C51E3) or plasmid subclones (KP\#451 or 452) carrying CELPC2 corrected both the egg-laying, body touch sensitivity, and the glr-1 suppression phenotypes of egl3(nu349) mutants (Table 1; also see egg-laying results below). All egl-3 alleles corresponded to missense mutations in predicted exons of CELPC2 (Fig. 3). With a single exception [n588 (E117Q)], all egl-3 alleles corresponded to changes in residues that are strongly conserved among proprotein convertases, suggesting that these alleles are likely to disrupt the function of the CELPC2 convertase. To identify a null allele of CELPC2, a PCR-based screen was used to isolate an in-frame deletion of 227 amino acids ( $n r 2090)$. The $n r 2090$ mutation is likely a null allele, because most of the catalytic domain is deleted in the mutant protein (Fig. 3); however, $n r 2090$ does not eliminate expression of the CELPC2 protein, as $n r 2090$ mutants still stain with antiCELPC2 antibodies (data not shown). The egg-laying, body touch sensitivity, and glr-1 suppression phenotypes of $n r 2090$ mutants were similar to those observed in nu349 mutants (Fig. 2; also see egg-laying results below). These results demonstrate that the egl-3 genetic locus corresponds to the CELPC2 gene. Hereafter, we refer to this gene as egl-3 PC2.

The egl-3 PC2 cDNA structure (Gomez-Saladin et al., 1994) predicts a protein that is $57 \%$ identical to human PC2. The predicted egl-3 PC2 protein contains all of the salient features of PC2, including a propeptide domain, a subtilisin-like catalytic domain (containing all of the predicted catalytic residues), a $\mathrm{P}$ domain, and dibasic residues for autocatalytic processing (Fig. 3). In addition, sequences within PC2 that are required for binding to the molecular chaperone 7B2 are conserved in egl-3 PC2, and a $C$. elegans 7B2 homolog has been identified (Lindberg et al., 1998).

\section{egl-3 PC2 is expressed broadly in the nervous system}

The expression pattern of egl-3 PC2 was determined by staining with anti-egl-3 PC2 antibodies (Fig. 4). Many neurons in both the head (mean, 46.6 head cells per animal) and tail ganglia (mean, 12 tail cells per animal) were stained with the anti-egl-3 PC2 antibodies (Fig. 4A). Because so many cells expressed egl-3 PC2, we did not attempt to comprehensively identify all expressing neurons. A subset of expressing cells were identified, including: the mechanosensory neurons ASH, ALM, AVM, and PVM; the interneurons AVB, AVD, PVC, RIG, and SDQL; and the HSN egg-laying motorneurons. Expression in the command interneurons AVB, AVD, and PVC was confirmed by double staining with anti-egl-3 PC2 and anti-glr-1 GluR antibodies (Fig. 4B). In addition, many motor neurons in the ventral nerve cord (mean, 37 cells per animal) were stained with anti-egl-3 PC2 antibody (Fig. $4 C$ ). Non-neural cell types (e.g., hypodermis and muscle cells) were not stained with the anti-egl-3 PC2 antibody. These results are consistent with previous reports characterizing the expression of the CELPC2 gene (Gomez-Saladin et al., 1994; Zahn et al., 2001).

Axon bundles in the nerve ring and ventral nerve cords of wild-type animals were brightly stained with the anti-egl-3 PC2 antibody (Fig. 4D). Staining of axons with anti-egl-3 PC2 antibodies could correspond to PC2 that has been trafficked into the secretory pathway. To test this possibility, we examined the subcellular distribution of endogenously expressed egl-3 PC2 protein in homozygous nu349 mutants. Catalytically inactive forms of human PC1 and mouse PC2 are inefficiently trafficked from the Golgi complex to secretory granules (Furuta et al., 1997; Jackson et al., 1997). Unlike wild-type animals, in egl-3(nu349) mutants, the anti-egl-3 PC2 staining was restricted to neuron cell bodies, whereas nerve ring and ventral cord staining were greatly reduced (Fig. 4E). Similar results were also obtained with egl-3(nr2090) mutants (data not shown). Thus, proper enzymatic activity is required for trafficking of the egl-3 PC2 protein into axons, as would be predicted if axonal staining consists of PC2 that has been transported into post-Golgi secretory organelles. Because egl-3 PC2 is broadly expressed in the worm nervous system and is apparently localized to secretory organelles in axons, it is likely that egl-3 PC2 plays an important role in processing of endogenous peptide neuromodulators. We next examined the behavior of egl-3 PC2 mutants to determine the roles played by these neuromodulators in vivo.

\section{Role of egl-3 PC2 in egg laying}

Egg-laying defects in egl-3 PC2 mutants were first described in Trent et al. (1983). We have extended these studies by analyzing additional egl-3 PC2 alleles. Egg-laying defects are evidenced by the increased number of eggs retained in utero in mutant hermaphrodites: wild type retained $12 \pm 2$ eggs, whereas egl-3 PC2 mutants retained significantly more (nr2090 $39 \pm 1$, nu349 $34 \pm 1$, $n 150 t s 41 \pm 4)$. The egg-laying defect observed in egl-3(nu349) mutants was corrected by introducing a rescuing egl-3 PC2 transgene (KP\#451): transgenic animals retained only $13.7 \pm 0.9$ eggs. The nu349 allele failed to complement n150ts for this egg-laying defect: nu349/n150ts heterozygotes retained $34 \pm 2$ eggs. We found that the egg laying of egl-3 PC2 mutants was resistant to both serotonin and imipramine. Serotonin $(3 \mathrm{mg} / \mathrm{ml})$ or imipramine $(0.75 \mathrm{mg} / \mathrm{ml})$ treatment caused wild-type animals to lay a large fraction of their retained eggs (serotonin 78\%, imipramine $86 \%)$, whereas these treatments caused egl-3(nr2090) PC2 mutants to lay a smaller fraction of their eggs (serotonin $2.6 \%$, imipramine $7.7 \%$ ). Similar results were also observed for homozygous nu349and n150ts mutants (data not shown). Egg-laying muscles and the HSN neurons had normal anatomical positions and morphologies in egl-3 PC2 mutants, suggesting that the development of the egg-laying circuit is not grossly impaired in egl-3 PC2 mutants. These results are most consistent with the idea that the egl-3 PC2 is required for the egg-laying muscles to become responsive to serotonin, although further studies will be required to determine the precise role played by egl-3 PC2 in egg laying. 
Table 2. Effect of egl-3 mutations on sensory behaviors

\begin{tabular}{|c|c|c|c|c|}
\hline Genotype [gene product] & $\begin{array}{l}\text { Osmosensory response } \\
\text { (\% defective) }\end{array}$ & $\begin{array}{l}\text { Volatile avoidance reversal } \\
\text { time (seconds) }\end{array}$ & $\begin{array}{l}\text { Nose touch response } \\
\text { (\% responding) }\end{array}$ & $\begin{array}{l}\text { Body touch } \\
\text { response }\end{array}$ \\
\hline Wild type & $4 \pm 2$ & $2.8 \pm 0.5$ & $82 \pm 8$ & WT \\
\hline eat-4(n2474) [VGLUT] & $58 \pm 4$ & $14.4 \pm 1.0$ & $4 \pm 2$ & WT \\
\hline$g l r-1(n 2461)[\mathrm{GluR}]$ & $12 \pm 6$ & $7 \pm 2.0$ & $14 \pm 8$ & $\mathrm{WT}$ \\
\hline egl-3(nu349) [PC2] & $4 \pm 2$ & $2.2 \pm 0.3$ & $87 \pm 4$ & Mec \\
\hline glr-1(n2461);egl-3(nu349) & $7 \pm 1$ & $3.7 \pm 1.2$ & $88 \pm 5$ & $\mathrm{Mec}$ \\
\hline
\end{tabular}

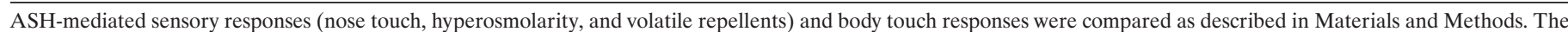

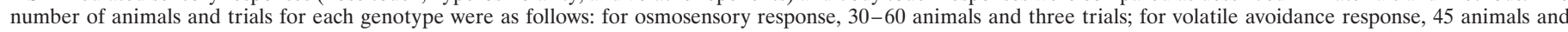

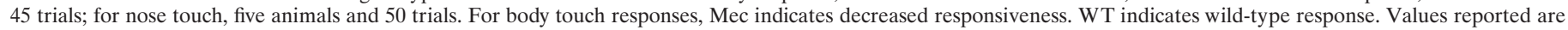
means \pm SEs.

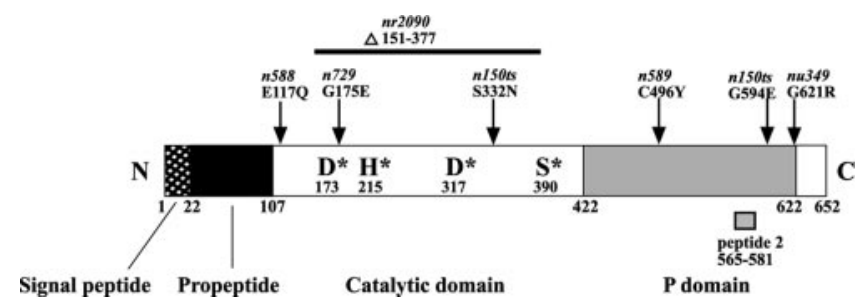

Figure 3. The structure of the egl-3 PC2 protein (as predicted from the cDNA sequence) is $57 \%$ identical to human PC2 and contains predicted propeptide, catalytic, and $\mathrm{P}$ domains. Within the catalytic domain, all four amino acid residues known to be critical for protease activity are conserved. Missense mutations in egl-3 PC2 alleles are indicated. The $n r 2090$ allele is an in-frame deletion that removes amino acids 151-377, as indicated. The synthetic peptide (peptide 2) sequence used to raise anti-egl-3 PC2 antisera is indicated by the gray bar.

\section{Restoration of the nose touch response requires functional ASH-command neuron synapses}

How does egl-3 PC2 regulate ASH-mediated nose touch sensitivity? The egl-3 PC2 mutations might bypass the requirement for ASH-command neuron synapses in the nose touch response. For example, in egl-3 PC2 mutants, another sensory neuron could be recruited to sense the touch stimulus, thereby eliminating the need for functional ASH-command neuron synapses. We tested this possibility by examining the touch sensitivity of $g l r-1$ GluR; egl-3 PC2 double mutants after killing the ASH neurons with a laser microbeam (Fig. 5A). We found that killing the ASH neurons greatly decreased the touch sensitivity of glr-1 GluR;egl-3 PC2 double mutants, demonstrating that restoration of the touch response required functional $\mathrm{ASH}$ neurons. These results suggest that the ASH-command neuron synapses were altered in egl-3 PC2 mutants such that they still function in the absence of glr-1 GluRs. Whatever modification of the ASH-command neuron synapses has occurred in egl-3 PC2 mutants does not grossly alter function of these synapses, because egl-3(nu349) single mutants responded normally to nose touch, hyperosmolarity, and to volatile repellents (Table 2).

A second potential mechanism for restoration of the nose touch response is that absence of egl-3 PC2 activity facilitates release of neurotransmitters other than glutamate in response to nose touch. In this case, the nose touch response might be less dependent on postsynaptic $g l r-1$ GluRs. We tested this possibility by examining the behavior of eat-4 VGLUT;egl-3 PC2 double mutants, which are defective for glutamate release (Lee et al., 1999; Bellochio et al., 2000; Takamori et al., 2000). We found that eat-4 VGLUT; egl-3 PC2 double mutants were insensitive to nose touch (Fig. $5 B$ ), suggesting that glutamate release is required for restoration of the nose touch response.

\section{Expression in the command neurons mediates the effects of egl-3 PC2 on nose touch sensitivity}

The effects of egl-3 PC2 mutations on the nose touch response could reflect its function in $\mathrm{ASH}$, the command neurons, or other cells. To determine where egl-3 PC2 expression is required for the proper function of ASH-command neuron synapses, we engineered expression vectors driving egl-3 PC2 expression in either ASH or the command neurons. Expression of egl-3 PC2 in ASH (using the osm-10 promoter) failed to rescue the $g l r-1$ GluR suppression defect, the body touch insensitivity, and the egglaying defects of glr-1 GluR;egl-3 PC2 double mutants (Fig. 5C) (data not shown). By contrast, expression of egl-3 PC2 in the command neurons (using the $g l r-1$ promoter) rescued the $g l r-1$ suppression defect, but not the egg-laying or body touch insensitivity defects of glr-1 GluR;egl-3 PC2 double mutants (Fig. 5C) (data not shown). These results suggest that expression in the command neurons mediates the effects of egl-3 PC2 on the nose touch response. Consistent with this idea, we found that egl-3 PC2 was expressed in the command neurons (Fig. 4B).

\section{DISCUSSION}

We have shown that the egl-3 PC2 gene plays a pivotal role in egg-laying behavior and in regulating the sensitivity of $C$. elegans to mechanosensory stimuli. We showed that mutations in egl-3 PC2 completely restored the nose touch response of glr-1 GluR mutants, suggesting that egl-3 $\mathrm{PC} 2$-processed peptides regulate the function of ASH-command neuron synapses. By contrast, mutants lacking egl-3 PC2 had diminished responsiveness to body touch stimuli. Our primary conclusion from these results is that the levels of egl-3 PC2 activity set the sensitivity of animals to two distinct touch stimuli.

Which egl-3 PC2 substrates are responsible for the behavioral defects we observed? The $C$. elegans genome sequence predicts the presence of at least 41 genes that encode potential neuropeptides, of which 20 are predicted to encode FMRFamide-related proteins (FaRPs), and 19 encode other neuropeptide-like proteins ( $\mathrm{Li}$ et al., 1999). In addition, $\sim 40$ genes are predicted to encode insulin-like proteins (Duret et al., 1998; Gregoire et al., 1998; Kawano et al., 2000). It is likely that this represents an underestimate of the number of potential egl-3 PC2 substrates, because genes encoding small peptides may have been missed by gene predicting algorithms.

One strategy for predicting egl-3 PC2 substrates is to compare the phenotype of egl-3 PC2 mutants with those of neuropeptide or neuropeptide receptor mutants. The $f p-1$ gene encodes 8 potential FaRPs (Nelson et al., 1998). Furthermore, the command neurons are stained with anti-FaRP antibodies (Schinkmann and Li, 1992), consistent with the idea that FaRPs might play a role in 

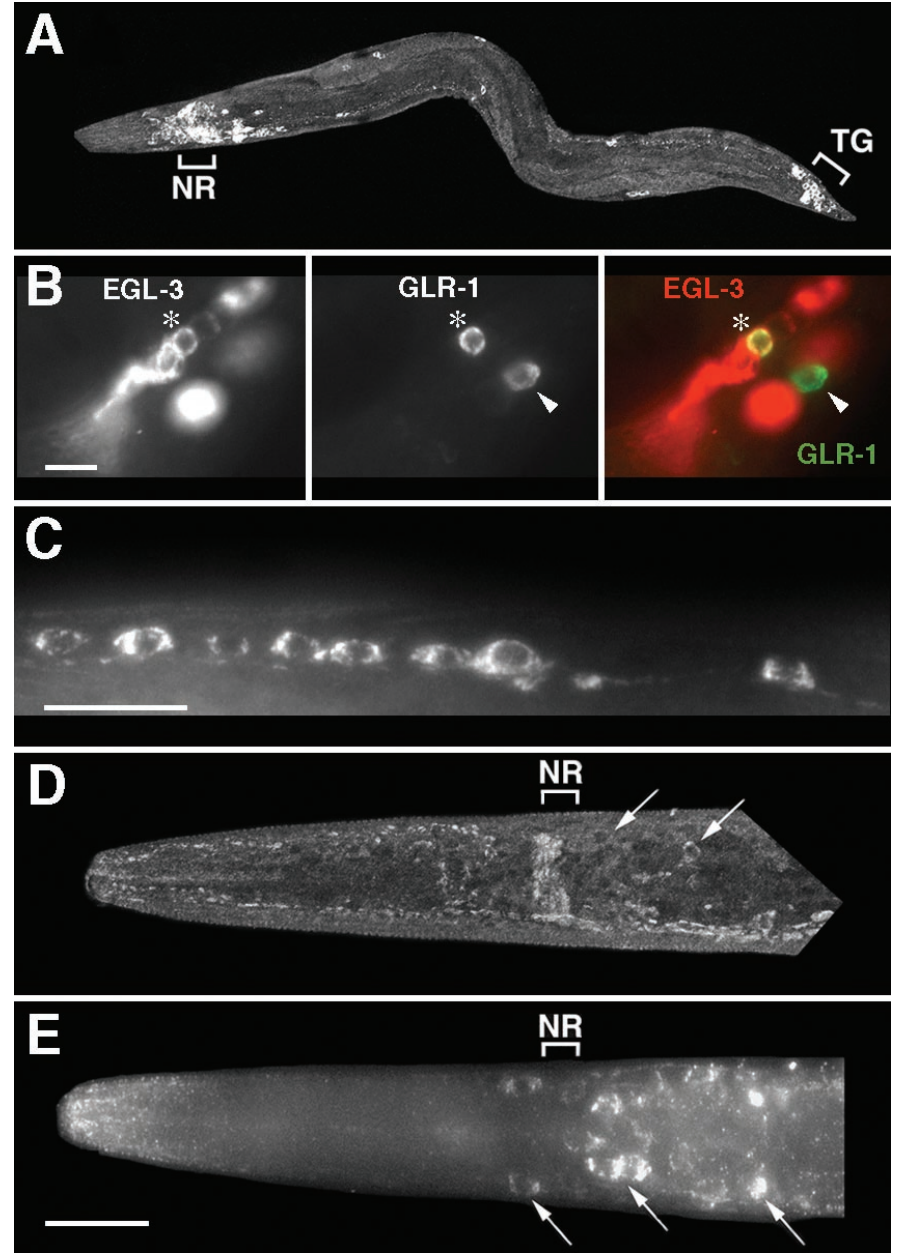

Figure 4. Expression of egl-3 PC2. Anti-egl-3 PC2 antibody was used to stain transgenic animals expressing KP\#454, a full-length rescuing EGL-3::GFP construct $(A-C)$. Endogenously expressed EGL-3 was visualized by staining nontransgenic wild-type animals $(D)$ or egl-3(nu349) mutants $(E)$. Expression of egl-3 PC2 in the command neurons was examined by staining transgenic animals containing both KP\#454 and nuIs24, a GLR-1::GFP transgene, with anti-egl-3 PC2 and anti-glr-1 GluR antibodies $(B) . A$, Expression of EGL-3::GFP was found in the cell bodies of many neurons in the head and in ganglia in the tail $(T G)$. In addition, axons in the nerve ring $(N R)$ stained brightly with the anti-egl-3 PC2 antibody. B, Expression of EGL-3::GFP in the PVC command neurons was documented by double staining with anti-egl-3 PC2 (left) and anti-glr-1 GluR (middle) antibodies. The merged image is shown on the right. This panel shows a mosaic animal in which one PVC neuron (indicated by the asterisk) expressed both GLR-1 (green) and EGL-3 (red), whereas the second PVC neuron (indicated by the arrowhead) expressed GLR-1 but not EGL-3. Similar results were obtained documenting expression of EGL-3::GFP in AVB and AVD (data not shown). $D, E$, Endogenously expressed EGL-3 was stained with anti-egl-3 PC2 antibodies. In wild-type animals $(D)$, bright staining was observed in the nerve ring $(N R)$ and ventral cord (data not shown) axons. Cell bodies (indicated by arrows) stained very weakly. In egl-3(nu349) mutants (E), the axons of the nerve ring $(N R)$ and ventral cord (data not shown) were poorly stained, whereas bright staining of neuronal cell bodies was observed (arrows). Scale bars, $10 \mu \mathrm{m}$.

regulating mechanosensory responsiveness. However, immunostaining with anti-FaRP antibodies (which detect only properly processed FaRP peptides) is superficially normal in egl-3 PC2 mutants (J. Kass, unpublished observations), suggesting that egl-3 PC2 is not required to process FaRPs. Mutations in a neuropeptide receptor homolog (NPR-1) cause $C$. elegans to forage in
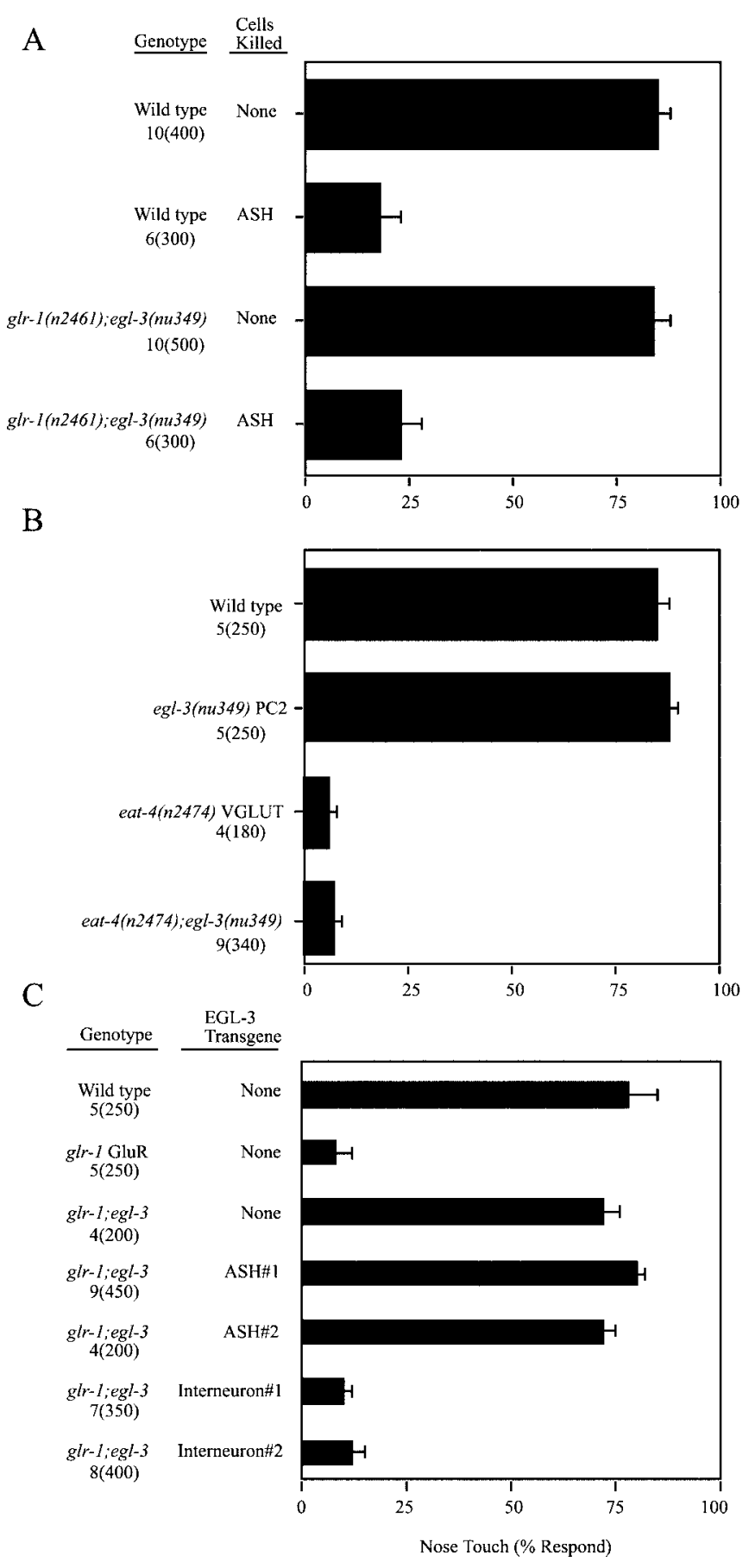

Figure 5. Role of egl-3 PC2 in restoration of the nose touch response. A, The nose touch responses of wild-type and of $g l r-1$ GluR;egl-3 PC2 double mutants are greatly diminished when the ASH neurons were killed with a laser microbeam. $B$, The egl-3(nu349) PC2 mutation did not restore nose touch responsiveness to eat-4 VGLUT mutants. $C$, egl-3 PC2 expression in the command neurons is sufficient to regulate ASH-mediated nose touch sensitivity. Wild-type EGL-3 was expressed in either the ASH neurons (using the $o s m-10$ promoter) or in the command neurons (using the $g l r-1$ promoter) of glr-1(n2461)GluR;egl-3(nu349)PC2 double mutants. For each transgene, two independent transgenic strains were analyzed. Strains expressing egl-3 PC2 in the command neurons (Interneuron\#1 and 2) had greatly reduced nose touch sensitivity, indicating rescue of the suppression defect. Rescue was not observed in transgenic strains expressing egl-3 PC2 in the ASH neurons ( $A S H \# 1$ and 2). Values shown are mean \pm SEs. The numbers listed below each genotype indicate the number of animals and nose touch trials for each data point, as follows: number of animals(number of trials). 
groups (de Bono and Bargmann, 1998), which is not observed in homozygous egl-3 PC2 mutants. Mutations in the insulin-like signaling pathway alter longevity and dauer formation (Kimura et al., 1997; Duret et al., 1998; Gregoire et al., 1998; Kawano et al., 2000). Homozygous egl-3 PC2 mutants do not have a dauer constitutive phenotype; however, insulin-like peptides have been implicated in both promoting and inhibiting dauer formation (Pierce et al., 2001). Therefore, our results do not exclude the possibility that egl-3 PC2 plays a role in processing endogenous insulin-like ligands. We conclude that the egl-3 PC2 behavioral defects are caused by the failure to synthesize a neuropeptide or neurohormone; however, the identity of the critical peptide or peptides is currently unknown.

The pronounced egg-laying and mechanosensory defects observed in egl-3 PC2 mutants suggests that neuropeptides or neurohormones play a critical role in these behaviors. Others have previously shown that FaRPs encoded by the $f l p-1$ gene alter the timing of egg-laying events (Waggoner et al., 2000); however, the egg-laying defects observed in egl-3 PC2 mutants are far more severe than those found in $f l p-1$ mutants (Nelson et al., 1998). Thus, it is likely egg-laying behavior is regulated by $f p-1$ FaRPs and by additional (currently unidentified) neuropeptides. Our analysis focused primarily on the role of egl-3 PC2 in mechanosensory responses.

How does egl-3 PC2 regulate mechanosensory responses? Neuropeptides and neurohormones often act as neuromodulators, regulating the impact of classical transmitters (Kupfermann, 1991). Neuropeptides can also act as trophic factors. However, we consider the latter an unlikely explanation for our results, because there was no obvious change in ASH or command neuron cell numbers or axon morphologies in egl-3 PC2 mutants (Kass, unpublished observations). Therefore, we favor the idea that changes in mechanosensory responsiveness in egl-3 PC2 mutants are caused by the absence of neuromodulatory peptides.

Homozygous egl-3 PC2 mutants have reciprocal defects in the nose and body touch responses. To explain the opposite effects of egl-3 PC2 mutations on these two responses, it is necessary to propose that the egl-3 $\mathrm{PC} 2$-processed peptides regulate synapses that are unique to each of these circuits (Fig. 1). In the case of the nose touch response, we propose that egl-3 PC2-processed peptides are released by the command neurons and that these peptides regulate the ASH-command neuron synapses. It is less likely that egl-3 PC2-processed peptides regulate the command neuronmotor neuron synapses, which are shared between the nose and body touch circuits. In the case of the body touch response, we propose that egl-3 PC2 acts in the touch neurons, which we have shown to express this convertase. Function in the command neurons is less likely in this case, because expressing wild-type egl-3 PC2 in the command neurons did not correct the body touch insensitivity defect of the egl-3 PC2 mutants.

Peptides released from the command neurons might inhibit nose touch-evoked ASH-command neuron neurotransmission by either of two sorts of mechanisms. Peptides could inhibit stimulus-evoked glutamate release from ASH. Alternatively, peptides might inhibit the response of command neurons to glutamate released from ASH. In either of these scenarios, ASHcommand neuron synapses might be more efficacious in egl-3 PC2 mutants, thereby increasing ASH-mediated nose touch sensitivity. Further experiments are required to distinguish between these possibilities.

Regulation of mechanosensory responses by egl-3 PC2processed peptides could provide a mechanism for C. elegans to modulate its locomotion in response to a change in ambient conditions. Different patterns of electrical activity are typically required to evoke release of neuropeptides than are required to release classical neurotransmitters. In general, neuropeptide release occurs only after high-frequency presynaptic activity or after more extreme membrane depolarization (Whim and Lloyd, 1989; Peng and Horn, 1991). Thus, we would predict that trains of touch stimuli or particularly intense stimuli would lead to the neuropeptide-induced decrease in mechanosensitivity. One attractive possibility is that neuropeptide release is involved in habituation of the nose touch response. We previously showed that responsiveness to nose touch is diminished by a train of 40 nose touch stimuli, whereas single touch stimuli do not alter sensitivity (Hart et al., 1999). We speculate that trains of touch stimuli are able to evoke neuropeptide release from the interneurons, which could contribute to the decreased sensitivity to further touch stimuli. Further experiments are required to test the potential connection between neuropeptides and habituation of the nose touch response.

In summary, we propose that unidentified neuropeptides regulate mechanosensory responses in C. elegans. Others have previously shown that neuropeptides regulate sensitivity of mice to painf ul stimuli. Knock-out mice lacking either substance $\mathrm{P}$ or its receptor have normal sensitivity to low-intensity noxious stimuli, but are less responsive to moderate noxious stimuli (Cao et al., 1998; De Felipe et al., 1998). Although the precise mechanisms involved may differ, our results suggest that $C$. elegans will provide a useful genetic model for studying the molecular mechanisms by which neuropeptides modulate sensory responses.

\section{REFERENCES}

Bargmann CI, Thomas JH, Horvitz HR (1990) Chemosensory cell function in the behavior and development of Caenorhabditis elegans. Cold Spring Harb Symp Quant Biol 55:529-538.

Bellochio E, Reimer R, Fremeau R, Edwards R (2000) Uptake of glutamate into synaptic vesicles by an inorganic phosphate transporter. Science 289:957-960.

Berger A, Hart A, Kaplan J (1998) G alphas-induced neurodegeneration in Caenorhabditis elegans. J Neurosci 18:2871-2880.

Besson J-M, Chaouch A (1987) Peripheral and spinal mechanisms of nociception. Phys Rev 67:67-186.

Cao YQ, Mantyh PW, Carlson EJ, Gillespie AM, Epstein CJ, Basbaum AI (1998) Primary afferent tachykinins are required to experience moderate to intense pain. Nature 392:390-394

Chalfie M, Au M (1989) Genetic control of differentiation of the $C$. elegans touch receptor neurons. Science 243:1027-1033.

Chalfie M, Sulston JE, White JG, Southgate E, Thomson JN, Brenner S (1985) The neural circuit for touch sensitivity in C. elegans. J Neurosci 5:956-964.

Creemers JW, Jackson RS, Hutton JC (1998) Molecular and cellular regulation of prohormone processing. Semin Cell Dev Biol 9:3-10.

de Bono M, Bargmann CI (1998) Natural variation in a neuropeptide Y receptor homolog modifies social behavior and food response in $C$. elegans. Cell 94:679-689.

De Felipe C, Herrero JF, O'Brien JA, Palmer JA, Doyle CA, Smith AJ, Laird JM, Belmonte C, Cervero F, Hunt SP (1998) Altered nociception, analgesia and aggression in mice lacking the receptor for substance P. Nature 392:394-397.

Driscoll M, Kaplan J (1997) Mechanotransduction. In: C. elegans II (Riddle D, Blumenthal T, Meyer B, Priess J, eds), pp 645-677. Cold Spring Harbor, NY: Cold Spring Harbor.

Duret L, Guex N, Peitsch MC, Bairoch A (1998) New insulin-like proteins with atypical disulfide bond pattern characterized in Caenorhabditis elegans by comparative sequence analysis and homology modeling. Genome Res 8:348-353.

Furuta M, Yano H, Zhou A, Rouille Y, Holst JJ, Carroll R, Ravazzola M, Orci L, Furuta H, Steiner DF (1997) Defective prohormone processing and altered pancreatic islet morphology in mice lacking active SPC2. Proc Natl Acad Sci USA 94:6646-6651.

Gomez-Saladin E, Wilson DL, Dickerson IM (1994) Isolation and in situ localization of a cDNA encoding a Kex2-like prohormone convertase in the nematode Caenorhabditis elegans. Cell Mol Neurobiol 14:9-25.

Gregoire FM, Chomiki N, Kachinskas D, Warden CH (1998) Cloning 
and developmental regulation of a novel member of the insulin- like gene family in Caenorhabditis elegans. Biochem Biophys Res Commun 249:385-390.

Harfe B, Fire A (1998) Muscle and nerve-specific regulation of a novel NK-2 class homeodomain factor in Caenorhabditis elegans. Dev Suppl 125:421-429.

Hart A, Sims S, Kaplan J (1995) A synaptic code for sensory modalities revealed by analysis of the $C$. elegans GLR-1 glutamate receptor. Nature 378:82-85

Hart AC, Kass J, Shapiro JE, Kaplan JM (1999) Distinct signaling pathways mediate touch and osmosensory responses in a polymodal sensory neuron. J Neurosci 19:1952-1958.

Jackson RS, Creemers JW, Ohagi S, Raffin-Sanson ML, Sanders L, Montague CT, Hutton JC, O'Rahilly S (1997) Obesity and impaired prohormone processing associated with mutations in the human prohormone convertase 1 gene. Nat Genet 16:303-306.

Kaplan JM, Horvitz HR (1993) A dual mechanosensory and chemosensory neuron in C. elegans. Proc Natl Acad Sci USA 90:2227-2231.

Kawano T, Ito Y, Ishiguro M, Takuwa K, Nakajima T, Kimura Y (2000) Molecular cloning and characterization of a new Insulin/IGF-like peptide of the nematode Caenorhabditis elegans. Biochem Biophys Res Commun 273:431-436.

Kimura KD, Tissenbaum HA, Liu Y, Ruvkun G (1997) daf-2, an insulin receptor-like gene that regulates longevity and diapause in Caenorhabditis elegans. Science 277:942-946.

Kupfermann I (1991) Functional studies of co-transmission. Physiol Rev 71:683-732.

Lee RYN, Sawin ER, Chalfie M, Horvitz HR, Avery L (1999) EAT-4, a homolog of a mammalian sodium-dependent inorganic phosphate cotransporter, is necessary for glutamatergic neurotransmission in Caenorhabditis elegans. J Neurosci 19:159-167.

Li C, Nelson LS, Kim K, Nathoo A, Hart AC (1999) Neuropeptide gene families in the nematode Caenorhabditis elegans. Ann NY Acad Sci 897:239-252.

Lindberg I, Tu B, Muller L, Dickerson IM (1998) Cloning and functional analysis of $C$. elegans 7B2. DNA Cell Biol 17:727-734.

Liu LX, Spoerke JM, Mulligan EL, Chen J, Reardon B, Westlund B, Sun L, Abel K, Armstrong B, Hardiman G, King J, McCague L, Basson M, Clover R, Johnson CD (1999) High-throughput isolation of Caenorhabditis elegans deletion mutants. Genome Res 9:859-867.

Maricq AV, Peckol E, Driscoll M, Bargmann C (1995) glr-1, a C. elegans glutamate receptor that mediates mechanosensory signaling. Nature 378:78-81.

Nelson LS, Rosoff ML, Li C (1998) Disruption of a neuropeptide gene, flp-1, causes multiple behavioral defects in Caenorhabditis elegans. Science 281:1686-1690.

Nonet M, Staunton J, Kilgard M, Fergestad T, Hartwieg E, Horvitz H, Jorgensen E, Meyer B (1997) Caenorhabditis elegans rab-3 mutant synapses exhibit impaired function and are partially depleted of vesicles. J Neurosci 17:8061-8073.
Peng YY, Horn JP (1991) Continuous repetitive stimuli are more effective than bursts for evoking LHRH release in bullfrog sympathetic ganglia. J Neurosci 11:85-95.

Perkins LA, Hedgecock EM, Thomson JN, Culotti JG (1986) Mutant sensory cilia in the nematode Caenorhabditis elegans. Dev Biol 117:456-487.

Pierce SB, Costa M, Wisotzkey R, Devadhar S, Homburger SA, Buchman AR, Ferguson KC, Heller J, Platt DM, Pasquinelli AA, Liu LX, Doberstein SK, Ruvkun G (2001) Regulation of DAF-2 receptor signaling by human insulin and ins-1, a member of the unusually large and diverse $C$. elegans insulin gene family. Genes Dev 15:672-686.

Rongo C, Whitfield CW, Rodal A, Kim SK, Kaplan JM (1998) LIN-10 is a shared component of the polarized protein localization pathways in neurons and epithelia. Cell 94:751-759.

Schinkmann K, Li C (1992) Localization of FMRF-amide-like peptides in C. elegans. J Comp Neurol 316:251-260.

Siekhaus DE, Fuller RS (1999) A role for amontillado, the Drosophila homolog of the neuropeptide precursor processing protease PC2, in triggering hatching behavior. J Neurosci 19:6942-6954.

Steiner DF (1998) The proprotein convertases. Curr Opin Chem Biol 2:31-39.

Takamori S, Rhee JS, Rosenmund C, Jahn R (2000) Identification of a vesicular glutamate transporter that defines a glutamatergic phenotype in neurons. Nature 407:189-194.

Trent C, Tsung N, Horvitz HR (1983) Egg-laying defective mutants of the nematode Caenorhabditis elegans. Genetics 104:619-647.

Troemel E, Chou H, Dwyer N, Colbert H, Bargmann C (1995) Divergent seven transmembrane receptors are candidate chemosensory receptors in C. elegans. Cell 83:207-218.

Waggoner LE, Hardaker LA, Golik S, Schafer WR (2000) Effect of a neuropeptide gene on behavioral states in Caenorhabditis elegans egglaying. Genetics 154:1181-1192.

Westphal CH, Muller L, Zhou A, Zhu X, Bonner-Weir S, Schambelan M, Steiner DF, Lindberg I, Leder P (1999) The neuroendocrine protein $7 \mathrm{~B} 2$ is required for peptide hormone processing in vivo and provides a novel mechanism for pituitary Cushing's disease. Cell 96:689-700.

Whim M, Lloyd P (1989) Frequency-dependent release of peptide cotransmitters from identified cholinergic motor neurons in Aplysia. Proc Natl Acad Sci USA 86:9034-9038.

Wicks SR, Rankin CH (1995) Integration of mechanosensory stimuli in C. elegans. J Neurosci 15:2434-2444.

Wicks SR, Roehrig CJ, Rankin CH (1996) A dynamic network simulation of the nematode tap withdrawal circuit: predictions concerning synaptic function using behavioral criteria. J Neurosci 16:4017-4031.

Zahn TR, Macmorris MA, Dong W, Day R, Hutton JC (2001) IDA-1, a Caenorhabditis elegans homolog of the diabetic autoantigens IA- 2 and phogrin, is expressed in peptidergic neurons in the worm. J Comp Neurol 429:127-143. 